\title{
氢气氛退火对硅上低温外延制备的硅锗薄膜性能的影响
}

\author{
王锦 ${ }^{1}$, 陶 科 ${ }^{2}$, 李国峰 ${ }^{1}$, 梁 科 ${ }^{1}$, 蔡宏琨 ${ }^{1}$ \\ (1. 南开大学 电子信息与光学工程学院, 天津 $300071 ; 2$. 中国科学院 微电子研究所, 北京 100029)
}

摘 要: 采用反应型热化学气相沉积系统在硅(100)衬底上外延生长富锗硅锗薄膜。四氟化锗作为锗源, 乙硅烷作为 还原性气体。通过设计表面反应，在低温条件下 $\left(350^{\circ} \mathrm{C}\right)$ 制备了高质量的富锗硅锗薄膜。研究了氢退火对低温硅锗 外延薄膜微结构和电学性能的影响。结果发现退火温度高于 $700^{\circ} \mathrm{C}$ 时, 外延薄膜的表面形貌随着退火温度的升高迅速 恶化。当退火温度为 $650^{\circ} \mathrm{C}$ 时, 获得了最佳的退火效果。在该退火条件下, 外延薄膜的螺旋位错密度从 $3.7 \times 10^{6} \mathrm{~cm}^{-2}$ 下降到 $4.3 \times 10^{5} \mathrm{~cm}^{-2}$, 表面粗粘度从 $1.27 \mathrm{~nm}$ 下降到 $1.18 \mathrm{~nm}$, 而外延薄膜的结晶质量也有效提高。霍尔效应测试表 明, 经退火处理的样品载流子迁移率明显提高。这些结果表明, 经过氢退火处理后, 反应型热化学气相沉积制备的 低温硅锗外延薄膜可以获得与高温下硅锗外延薄膜相比拟的性能。

关 键 词: 硅锗薄膜; 低温外延; 氢退火; 螺旋位错

中图分类号: TQ174 文献标识码: A

\section{Effect of Hydrogen Annealing on the Property of Low-temperature Epitaxial Growth of Sige Thin Films on Si Substrate}

\begin{abstract}
WANG Jin ${ }^{1}$, TAO Ke ${ }^{2}$, LI Guo-Feng ${ }^{1}$, LIANG Ke ${ }^{1}$, CAI Hong-Kun ${ }^{1}$
(1. College of Electronic Information and Optical Engineering, Nankai University, Tianjin 300071, China; 2. Institute of Microelectronics, Chinese Academy of Sciences, Beijing 100029, China)

Abstract: Ge-rich SiGe thin films were epitaxially grown on Si (100) substrate by reactive thermal chemical vapor deposition. Germanium tetrafluoride $\left(\mathrm{GeF}_{4}\right)$ was selected as the Ge source material and disilane $\left(\mathrm{Si}_{2} \mathrm{H}_{6}\right)$ is used as the reductant gas. By designing the surface reaction, high quality Ge-rich $\mathrm{Si}_{1-x} \mathrm{Ge}_{x}$ epilayers were prepared under low temperature $\left(350^{\circ} \mathrm{C}\right)$ conditions. Effect of hydrogen annealing on the microstructure and electrical property was studied. The results reveal that the surface morphology of SiGe epilayer deteriorates drastically if the annealing temperature is higher than $700^{\circ} \mathrm{C}$. The optimal annealing temperature is found to be $650^{\circ} \mathrm{C}$. Under this condition, the threading dislocation density decreases from $3.7 \times 10^{6} \mathrm{~cm}^{-2}$ to $4.3 \times 10^{5} \mathrm{~cm}^{-2}$ and the surface root mean square roughness is also slightly decreased from $1.27 \mathrm{~nm}$ to $1.18 \mathrm{~nm}$. Simultaneously, the crystalline quality of the SiGe epilayers is effectively improved. Carrier mobility of the annealed samples, measured by Hall-effect equipment, is enhanced obviously as compared to that of the as-deposited samples. These results suggest that the properties of the annealed SiGe thin films, epitaxially grown by reactive thermal chemical vapor deposition at low-temperature, can be comparable to those of SiGe epilayers grown by high-temperature techniques.
\end{abstract}

Key words: silicon germanium; low-temperature epitaxy; hydrogen annealing; threading dislocation density

近年来，硅锗合金因其在光电子器件方面的应用潜力吸引了广泛关注。一方面，硅锗材料的载流 
子迁移率高、能带宽度随锗组分的变化连续可调, 且与目前成熟的硅平面工艺相兼容; 另一方面, 在 太阳光谱近红外波段吸收系数高。这些特点使得硅 锗材料可被应用到调制掺杂场效应晶体管、光电探 测器及太阳电池中 ${ }^{[1-4]}$ 。硅基祄底上异质外延硅锗薄 膜最大的挑战是硅锗之间存在 $4.18 \%$ 的晶格失配。 这种晶格失配不仅使硅锗外延薄膜表面粗粘, 而且 会形成大量的位错缺陷，造成载流子迁移率下降 ${ }^{[5]}$ 。 为了有效控制表面粗楉度并降低位错密度, 人们开 发了多种缓冲层技术, 其中, 组分缓变 $\mathrm{SiGe}$ 缓冲层 技术及低温缓冲层并结合退火处理技术(低温/高温 两步法)可以有效降低外延薄膜的螺旋位错密度和 表面粗粘度, 进而获得高质量硅锗外延薄膜 ${ }^{[6-11]}$ 。这 些技术主要基于超高真空化学气相沉积 (UHVCVD)、低压化学气相沉积(LP-CVD)和减压化学气 相沉积(RP-CVD) 等, 通常以硅烷和锗烷作为源气 体, 通过热分解来实现外延生长。所以源气体的热 分解温度是决定外延生长温度的主要因素。为了获 得高质量外延硅锗薄膜, 上述沉积技术所需的温度 都较高 $\left(>500^{\circ} \mathrm{C}\right)$ 。

本工作采用反应型热化学气相沉积系统, 以四 氟化锗和乙硅烷为源气体在硅基衬底上外延生长富 锗硅锗薄膜。四氟化锗的热分解温度高于 $1000^{\circ} \mathrm{C}$, 而 通过设计氧化还原反应可以将沉积温度降至 $300^{\circ} \mathrm{C}^{[12-15]}$ 。 氢退火是提高硅锗外延薄膜结晶质量的有效途径 ${ }^{[16-18]}$, 而对于低温生长的硅锗外延薄膜, 氢退火的效果研 究得很少。本工作在 $350^{\circ} \mathrm{C}$ 下制备硅锗外延薄膜, 研 究了氢退火对硅锗外延薄膜微结构的影响, 获得了 最佳的退火温度范围。

\section{1 实验方法}

在反应型热化学气相沉积系统中生长富锗硅锗 外延薄膜, 采用 $\phi 15 \mathrm{~cm}$ 的 $\mathrm{N}$ 型(100)单晶硅衬底(电 阻率 $\geqslant 1000 \Omega \cdot \mathrm{cm})$ 。采用 $\mathrm{RCA}$ 标准清洗工艺清洗硅 祄底, 在放入沉积系统前, 祄底在浓度为 $1 \%$ 氢氟酸 溶液中浸泡 $1 \mathrm{~min}$, 去除祄底表面的自然氧化物。沉 积室本底真空达到 $3.99 \times 10^{-4} \mathrm{~Pa}$ 后, 开始给祄底加 热。乙硅烷和四氟化锗作为源气体, 氦气作为载气, 通过喷头导入反应室。反应气压固定在 $1333 \mathrm{~Pa}, 乙$ 硅烷与四氟化锗的流量比固定在 1.5 , 衬底温度固 定在 $350^{\circ} \mathrm{C}$ 。当硅锗薄膜外延生长结束后, 自然冷却 到室温, 然后置入退火仪中进行氢气退火处理。当 退火仪本底真空低于 $3.99 \times 10^{-4} \mathrm{~Pa}$ 时, 开启加热系统, 祄底升温速率为 $3^{\circ} \mathrm{C} / \mathrm{s}$ 。氢气气压设定为 $11305 \mathrm{~Pa}$, 退
火温度在 $600 \sim 750^{\circ} \mathrm{C}$ 之间变动, 退火时间固定在 $20 \mathrm{~min}$ 。

采用扫描电镜(SEM: JSM-610LA)测定硅锗外 延薄膜的厚度，采用 AFM 观察薄膜的表面形貌。用 高分辨率 XRD(HR-XRD) 和拉曼谱及透射电镜(TEM: JEM-2010F) 分析硅锗外延薄膜的微结构。硅锗外延 薄膜中的螺旋位错密度由选择性湿法腐蚀技术并 结合扫描电镜来测定，最后通过计算样品表面的 腐蚀坑数量来衡量螺旋位错密度 ${ }^{[4,19]}$ 。硅锗外延薄 膜中的各元素深度分布由飞行时间-二次离子质谱 仪 (TOF-SIMS) 测定, 其中, Cs 离子束的能量为 $2000.0 \mathrm{eV}$, 溅射面积和分析面积分别是 $300 \mu \mathrm{m} \times$ $300 \mu \mathrm{m}$ 和 $100 \mu \mathrm{m} \times 100 \mu \mathrm{m}$ 。用霍尔仪在室温下测量 硅锗外延薄膜的电学特性(包括导电类型、电阻率、 载流子迁移率及浓度)。

\section{2 结果与讨论}

文献[15]介绍了反应型热化学气相沉积系统低 温外延硅锗薄膜的基本原理及特征，并通过优化沉 积工艺在 $350^{\circ} \mathrm{C}$ 获得了高质量的硅锗外延薄膜。其 中，锗的组分高达 $99 \%$, 薄膜中的应力弛豫度达到 $98.5 \%$ 。本工作首先通过控制薄膜的生长时间, 获得 了一组不同厚度的样品。图 1(a)给出了一个初始沉积 样品的断面透射电镜照片, 其中样品厚度约 $1.25 \mu \mathrm{m}$, 表面均方根粗糙度为 $1.27 \mathrm{~nm}(\mathrm{AFM})$ 。从图 1(a)中可 以看到, 除了极少数失配位错缺陷随着薄膜生长延 伸到硅锗外延薄膜中外，几乎所有的失配位错缺陷 都被限制在外延薄膜与硅祄底的界面附近。图 1(b)、 (c)和(d)给出了一组电子衍射谱, 分别来自样品断面 的 $\mathrm{Si}$ 衬底、 $\mathrm{SiGe} / \mathrm{Si}$ 异质结面及 $\mathrm{SiGe}$ 外延薄膜。一 方面, 这三个位置的电子衍射图样体现出良好的均 一性，表明硅锗外延薄膜完美地延续了硅祄底的晶 格排列方向; 另一方面，由于硅与锗之间的晶格失 配, 从 $\mathrm{SiGe} / \mathrm{Si}$ 异质结处得到的电子衍射谱实际上 包含两套衍射谱, 是单晶硅与单晶锗电子衍射谱的 叠加。通过标定电子衍射谱, 可以获得外延薄膜的 晶带轴方向[011], 如图 1(e)所示, 进一步说明硅锗 外延薄膜沿着硅衬底(100)方向生长。

将上述样品在氢氛围下进行退火处理, 退火温 度分别为 $600^{\circ} \mathrm{C} 、 650^{\circ} \mathrm{C} 、 700^{\circ} \mathrm{C}$ 和 $750^{\circ} \mathrm{C}$ 。结果发现, 在不同温度下退火的样品呈现出迥然相异的表面形 貌。在 $650^{\circ} \mathrm{C}$ 及以下退火的样品仍然保持着与初始 样品相同的镜面光滑, 而在 $700^{\circ} \mathrm{C}$ 和 $750^{\circ} \mathrm{C}$ 下退火的 样品则表面变得粗粘。图 2 给出了不同温度退火样 品的表面扫描电镜照片, 从图中可以看见, 当退火温 


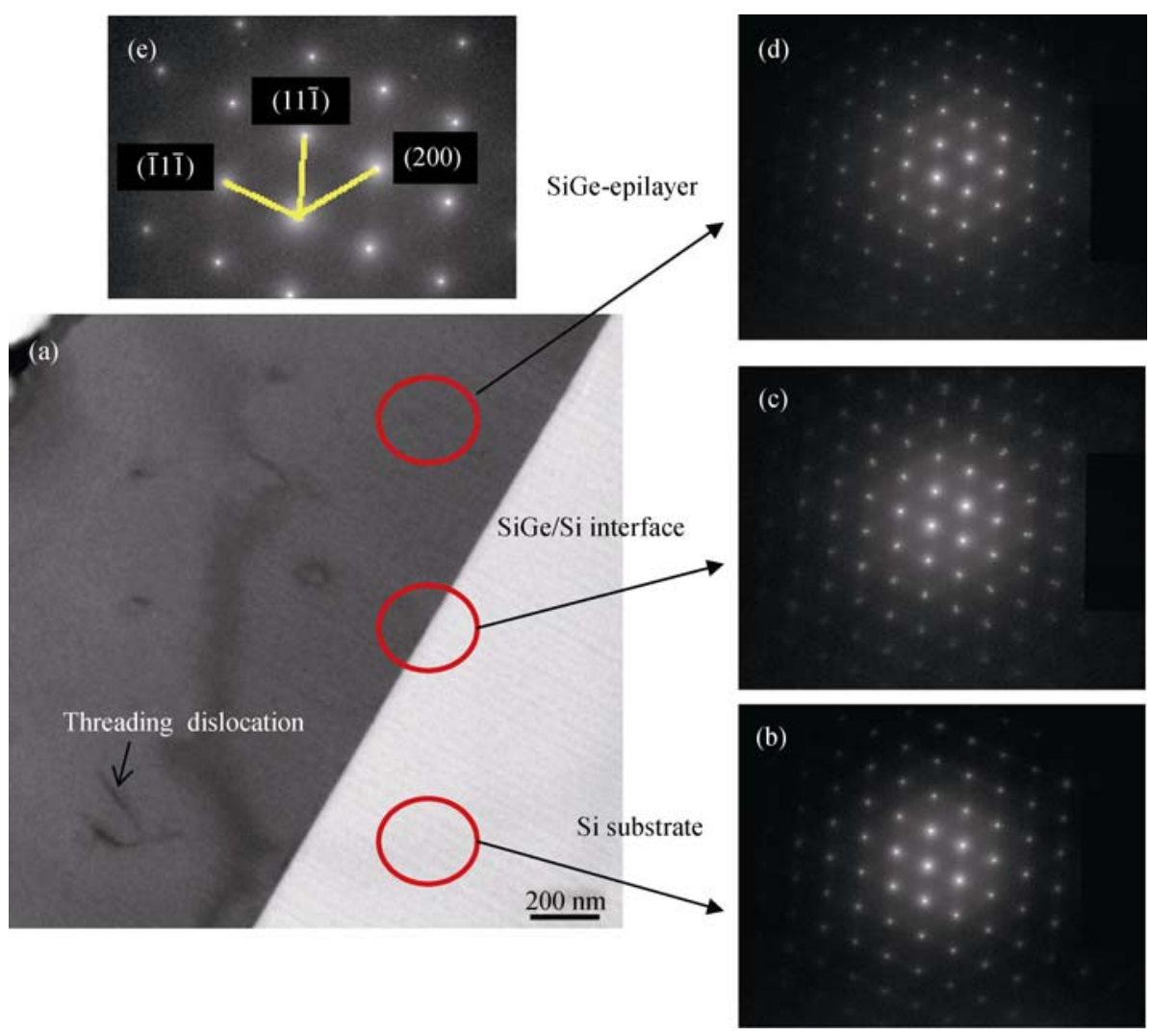

图 1 硅锗外延薄膜的断面透射电镜照片(a)及样品不同深度的电子衍射谱(b d)

Fig. 1 (a) Cross-sectional TEM image of epitaxial SiGe films on silicon substrate, (b)-(d) electron diffraction patterns for Si substrate, $\mathrm{SiGe} / \mathrm{Si}$ interface and $\mathrm{SiGe}$ epilayer (e) which extracted from (d) for calculation The red circles mark the position for measurement of electron diffraction patterns
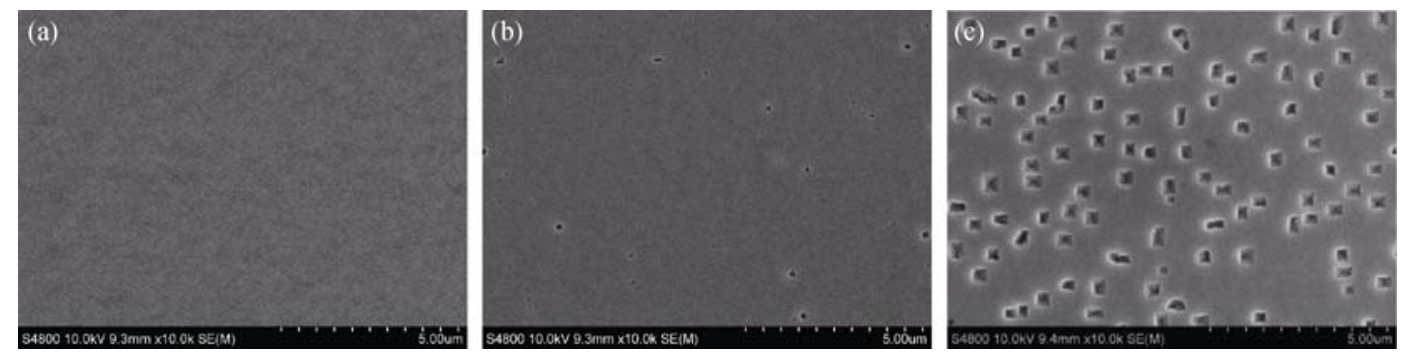

图 2 经不同温度退火后硅锗外延薄膜表面的 SEM 照片

Fig. 2 SEM images of SiGe epilayers annealed at different temperatures (a) $650^{\circ} \mathrm{C}$; (b) $700^{\circ} \mathrm{C}$; (c) $750^{\circ} \mathrm{C}$

度较高时, 样品的表面出现了一些“小坑”, 并且这 些“小坑”的密度及尺寸随着退火温度的升高而增 大。从图 2(c)可以观察到, 这些小坑呈现出倒金字 塔结构。这种结构的“小坑”与表征锗外延薄膜螺旋 位错密度所使用的选择性湿法腐蚀所得结果十分相 似, 由此推断, 这些倒金字塔结构的“小坑”也是腐 蚀所产生的结果。通过 AFM 测试发现, 硅锗外延薄 膜的表面均方根粗糙度随着退火温度的升高急剧上 升(从 $650^{\circ} \mathrm{C}$ 的 $1.18 \mathrm{~nm}$ 增大到 $750^{\circ} \mathrm{C}$ 的 $19.78 \mathrm{~nm}$ ), 说明退火温度过高将致使硅锗外延薄膜表面形貌恶 化, 从而不利于硅锗外延薄膜在器件上的应用。
分别测试了 $650^{\circ} \mathrm{C}$ 和 $700^{\circ} \mathrm{C}$ 退火处理样品的时 间飞行二次离子质谱, 以观察样品中硅、锗及氢、 硼、氧等杂质的深度分布。图 3(a)为 $650^{\circ} \mathrm{C}$ 退火样品 的 TOF-SIMS 测试结果, 测试样品的厚度约 $320 \mathrm{~nm}$ 。 在该温度下退火后，硅锗外延薄膜中的各成分的深 度分布非常稳定, 与初始生长的硅锗外延薄膜测试 结果相比, 退火样品体现出一个明显的特征, 就是 锗原子在硅祄底中的扩散加剧，这与 UHV- CVD、 RPCVD 和 LPCVD 等系统生长的硅锗外延薄膜所 得到的结果截然相反, 后者在退火过程中表现出 硅原子在硅锗外延薄膜中的扩散 ${ }^{[17]}$ 。图 3(b)给出 

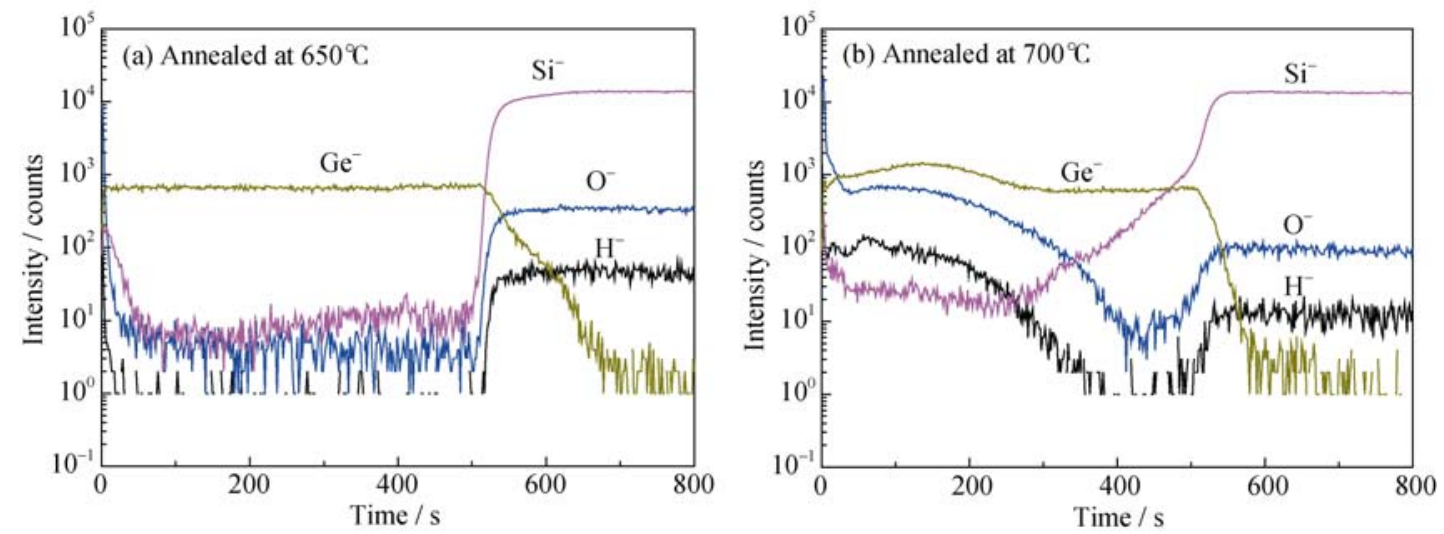

图 3 经不同温度退火处理后硅锗外延薄膜由 TOF-SIMS 测得的离子组分深度分布

Fig. 3 Composition depth profile of ions detected by TOF-SIMS measurement from epitaxial SiGe films annealed at different temperatures

了 $700^{\circ} \mathrm{C}$ 退火样品的 TOF-SIMS 测试结果。硅锗外延 薄膜的各成分随深度均发生较大的变化: 一方面, 大量硅原子自硅祄底向外延薄膜扩散, 使得外延薄 膜中锗的组分出现大幅下降; 另一方面, 外延薄膜 中氢原子及氧原子的含量比低温退火时明显升高, 在 表层浓度最高, 随探测深度的增加逐渐下降, 表现出 由表及里的扩散过程。显然, 在表面出现的倒金字塔 形“小坑”为氢、氧原子的内扩散提高了一条“捷径”。 对比图 3(a)和(b), 可以推断硅原子的外扩散存在一个 温度阈值, 当退火温度高于该阈值时, 硅原子在硅锗 外延薄膜中的扩散将大幅加剧, 而当退火温度低于该 阈值时, 硅原子由祄底向外延薄膜的扩散则较为微 弱。从实验结果不难得出, 该温度阈值处于 $650^{\circ} \mathrm{C}$ 和 $700^{\circ} \mathrm{C}$ 之间。而倒金字塔形“小坑”的形成似乎与氟原子 的外扩散有关, 具体原因尚在进一步研究之中。

上述实验结果表明, 当退火温度高于 $700^{\circ} \mathrm{C}$ 后, 硅锗外延薄膜的质量迅速下降, 接下来的实验将验 证 $650^{\circ} \mathrm{C}$ 为最佳退火温度。图 4 为初始外延薄膜及退 火后薄膜的拉曼谱。未经退火的样品除了在 $300 \mathrm{~cm}^{-1}$ 附近有一个很强的拉曼峰(对应于 Ge-GeTO 模)外, 在 $390 \mathrm{~cm}^{-1}$ 附近还有一个强度较弱, 半高宽(FWHM) 较大的拉曼峰(对应于 Ge-Si TO 模)。与之相比, 退 火处理后的硅锗外延薄膜拉曼谱 Ge-Ge TO 模不仅强 度增大, 半高宽也都显著下降(从 $6.368 \mathrm{~cm}^{-1}$ 降至 $4.850 \mathrm{~cm}^{-1}$ 以下), 还有一个明显的特征就是 $\mathrm{Ge}-\mathrm{Si}$ $\mathrm{TO}$ 模的变化。从图 4 可以看出, 退火样品 Ge-Si TO 模强度大幅减小, 而且峰位出现了蓝移。这表明退 火硅锗外延薄膜的结晶质量得到提高。比较 $600^{\circ} \mathrm{C}$ 退火的样品与 $650^{\circ} \mathrm{C}$ 退火的样品, 结果发现, $\mathrm{Ge}-\mathrm{Ge}$ $\mathrm{TO}$ 模半高宽随着退火温度的升高, 从 $4.850 \mathrm{~cm}^{-1}$ 降 到 $4.479 \mathrm{~cm}^{-1}$, 表明 $650^{\circ} \mathrm{C}$ 为最佳退火温度。需要指 出的是 Ge-Si TO 模峰位的蓝移与薄膜中硅锗组分

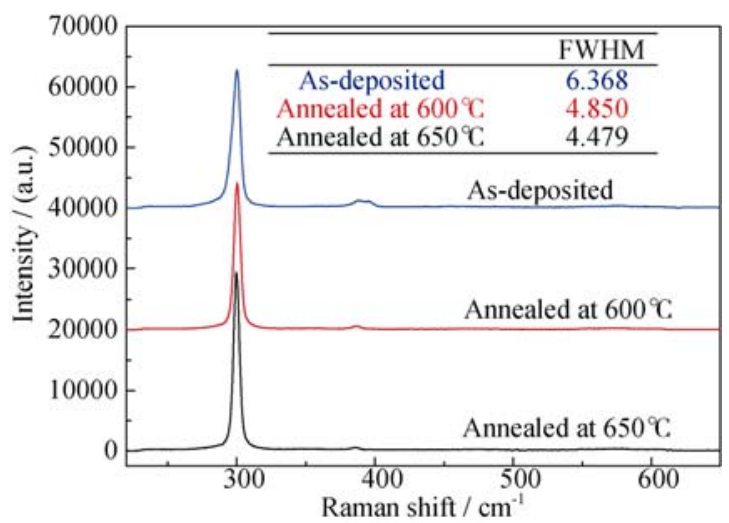

图 4 未经退火处理和经退火处理的硅锗外延薄膜的 Raman 谱图 Fig. 4 Raman spectra of epitaxial SiGe films with or without postannealing

变化或应力弛豫有关 ${ }^{[20]}$, 由于没有观察到硅原子向 外延薄膜扩散, 因此可以推断 Ge-Si TO 模峰位的蓝 移是外延薄膜中应力弛豫所致。

为了研究氢退火对硅锗外延薄膜中螺旋位错密 度的影响, 实验采用具有高灵敏度的湿法腐蚀技术 来反映薄膜中的螺旋位错。使用不含镉的酸性腐蚀液 (HF: $\mathrm{HNO}_{3}: \mathrm{CH}_{3} \mathrm{COOH}: \mathrm{I}_{2}=5 \mathrm{~mL}: 10 \mathrm{~mL}: 32 \mathrm{~mL}: 18 \mathrm{mg}$ ) 对硅锗外延薄膜进行腐蚀, 然后在扫描电镜下观察 腐蚀坑的数量, 从而得到螺旋位错密度, 如图 5 所 示。初始生长的外延薄膜和退火后的外延薄膜经酸 液腐蚀后表面均出现大量的腐蚀坑, 通过对样品 5 个不同位置的腐蚀坑密度进行标定，然后取平均值 作为该样品的螺旋位错密度。计算结果显示, 未退火 的外延薄膜, 其螺旋位错密度约为 $3.7 \times 10^{6} \mathrm{~cm}^{-2}$, 而 退火后外延薄膜的螺旋位错密度约为 $4.3 \times 10^{5} \mathrm{~cm}^{-2}$ 。 通过氢退火处理，可以使硅锗外延薄膜中的螺旋位 错密度降低约一个数量级。应用霍尔仪对硅锗外延 薄膜的电学特性进行了表征, 如表 1 所示。外延薄膜 

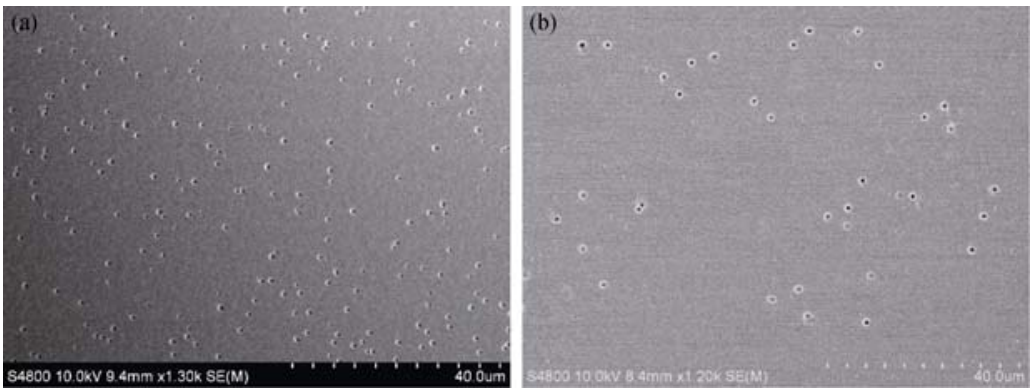

图 5 硅锗外延薄膜经选择性湿法腐蚀后的表面扫描电镜照片

Fig. 5 SEM images of SiGe films after a selective wet etch, and pits with reversed pyramidal structure exhibited on the surface (a) As-deposited sample; (b) $650^{\circ} \mathrm{C}$-annealed sample

表 1 由霍尔仪测得的硅锗外延薄膜的电学性能(膜厚 $900 \mathrm{~nm}$ )

Table 1 Electrical properties of SiGe thin films by Hall-effect measurement

\begin{tabular}{cccc}
\hline $\mathrm{Si}_{x} \mathrm{Ge}_{1-x}$ & $\begin{array}{c}\text { Resistance } \\
/(\Omega \cdot \mathrm{cm})\end{array}$ & $\begin{array}{c}\text { Carrier con- } \\
\text { centra- } \\
\text { tion } / \mathrm{cm}^{-3}\end{array}$ & $\begin{array}{c}\text { Mobility/ } \\
\left(\mathrm{cm}^{2} \cdot \mathrm{V}^{-1} \cdot \mathrm{s}^{-1}\right)\end{array}$ \\
\hline As-grown & 0.402 & $6.35 \times 10^{16}$ & 244 \\
Annealed & 1.470 & $1.07 \times 10^{16}$ & 409 \\
\hline
\end{tabular}

厚度为 $360 \mathrm{~nm}$, 呈 $\mathrm{P}$ 型导电。经过退火后, 外延薄膜 的本征载流子浓度由 $6.35 \times 10^{16} \mathrm{~cm}^{-3}$ 下降到 $1.07 \times$ $10^{16} \mathrm{~cm}^{-3}$, 而电阻率和载流子迁移率分别由 $0.402 \Omega \cdot \mathrm{cm}$ 和 $244 \mathrm{~cm}^{2} /(\mathrm{V} \cdot \mathrm{s})$ 增大到 $1.47 \Omega \cdot \mathrm{cm}$ 和 $409 \mathrm{~cm}^{2} /(\mathrm{V} \cdot \mathrm{s})$ 。 显然, 经过退火处理后, 薄膜的结晶质量进一步得 到改善, 缺陷密度下降, 进而使薄膜的电学特性得 到提高。氢气氛中退火, 使得外延薄膜表面形成 $\mathrm{Ge}-\mathrm{H}$ 键, 有效降低表面原子的扩散势垒 ${ }^{[16]}$, 促进 外延薄膜表面原子的迁移, 从而有利于表面原子形 成更有序的晶格结构。退火温度越高, 原子振动并 发生迁移的概率就越高, 对于改善薄膜的结晶质量
和电学特性将有更好的效果。但是退火温度过高, 例如超过 $700^{\circ} \mathrm{C}$, 大量硅原子则由硅祄底向外延薄 膜中扩散, 反而使外延薄膜性能变差。

表 2 列举了文献报道的采用低温/高温两步法制 备的锗外延薄膜的性能参数 ${ }^{[21-25]}$ 。对比以上结果, 可以发现采用反应型热化学气相沉积系统在 $350^{\circ} \mathrm{C}$ 外延生长的富锗硅锗薄膜经过 $650^{\circ} \mathrm{C}$ 氢退火处理后, 已经具有与高温条件下制备的硅锗外延薄膜相比拟 的结晶质量和电学性能, 说明本研究在低温制备高 质量硅锗外延薄膜方面具备较高的应用潜力。

\section{3 结论}

采用反应型热化学气相沉积在 $350^{\circ} \mathrm{C}$ 、硅 $(100)$ 祄底上外延生长富锗硅锗薄膜。研究了氢退火对低 温硅锗外延薄膜微结构和电学性能的影响。结果发 现，退火温度高于 $700^{\circ} \mathrm{C}$ 时，外延薄膜的表面形貌 随着退火温度的升高迅速恶化，且薄膜中碳、氢及 氧等杂质含量大幅升高。当退火温度为 $650^{\circ} \mathrm{C}$ 时, 获 得了最佳的退火效果，薄膜的表面粗粘度有所降低，

表 2 文献中报道的采用低温/高温法制备的锗外延薄膜的性能参数

Table 2 Summary of the process parameters and film quality from literatures which reported the epitaxial growth of Ge by using low temperature/high temperature method

\begin{tabular}{|c|c|c|c|c|c|c|c|c|}
\hline \multirow{2}{*}{ Ref. } & & \multirow{2}{*}{ Temp. $/{ }^{\circ} \mathrm{C}$} & \multirow{2}{*}{$\begin{array}{c}\text { Thickness } \\
/ \mathrm{nm}\end{array}$} & \multicolumn{2}{|c|}{ RMS roughness $/ \mathrm{nm}$} & \multicolumn{2}{|c|}{$\mathrm{TDD} / \mathrm{cm}^{-2}$} & \multirow{2}{*}{$\frac{\text { Mobility } /\left(\mathrm{cm}^{2} \cdot \mathrm{V}^{-1} \cdot \mathrm{s}^{-1}\right)}{\text { Annealed }}$} \\
\hline & & & & As-grown & Annealed & As-grown & Annealed & \\
\hline \multirow{2}{*}{ [21] } & LT & 400 & \multirow{2}{*}{1224} & \multirow{2}{*}{0.40} & \multirow{2}{*}{0.7} & \multirow{2}{*}{$1.70 \times 10^{8}$} & \multirow{2}{*}{$1.00 \times 10^{7}$} & \multirow{2}{*}{ NA } \\
\hline & HT & 670 & & & & & & \\
\hline \multirow{2}{*}{ [22] } & LT & 350 & 50 & \multirow{2}{*}{0.70} & \multirow{2}{*}{ NA } & \multirow{2}{*}{$5.00 \times 10^{5}$} & \multirow{2}{*}{ NA } & \multirow{2}{*}{550} \\
\hline & HT & 600 & 300 & & & & & \\
\hline \multirow{2}{*}{ [23] } & $\mathrm{LT}$ & 335 & \multirow{2}{*}{2000} & \multirow{2}{*}{0.60} & \multirow{2}{*}{1.6} & \multirow{2}{*}{ NA } & \multirow{2}{*}{$2.00 \times 10^{7}$} & \multirow{2}{*}{ NA } \\
\hline & HT & 670 & & & & & & \\
\hline \multirow{2}{*}{ [24] } & LT & 400 & \multirow{2}{*}{2500} & \multirow{2}{*}{1.20} & \multirow{2}{*}{1.0} & \multirow{2}{*}{ NA } & \multirow{2}{*}{$6.00 \times 10^{6}$} & \multirow{2}{*}{ NA } \\
\hline & HT & 750 & & & & & & \\
\hline \multirow{2}{*}[25]{} & $\mathrm{LT}$ & 400 & \multirow{2}{*}{980} & \multirow{2}{*}{3.19} & \multirow{2}{*}{0.9} & \multirow{2}{*}{ NA } & \multirow{2}{*}{$6.00 \times 10^{6}$} & NA \\
\hline & HT & 670 & & & & & & \\
\hline
\end{tabular}


结晶质量显著提高。在该退火条件下, 外延薄膜的 螺旋位错密度从 $3.7 \times 10^{6} \mathrm{~cm}^{-2}$ 下降到 $4.3 \times 10^{5} \mathrm{~cm}^{-2}$, 霍尔迁移率从 $244 \mathrm{~cm}^{2} /(\mathrm{V} \cdot \mathrm{s})$ 增大到 $409 \mathrm{~cm}^{2} /(\mathrm{V} \cdot \mathrm{s})$, 本征载流子浓度也由 $6.35 \times 10^{16} \mathrm{~cm}^{-3}$ 下降到 $1.07 \times$ $10^{16} \mathrm{~cm}^{-3}$ 。这些结果表明, 在 $350^{\circ} \mathrm{C}$ 外延生长的富锗 硅锗薄膜经过 $650^{\circ} \mathrm{C}$ 氢退火处理后，已经具有与高 温条件下制备的硅锗外延薄膜相比拟的结晶质量和 电学性能, 说明反应型热化学气相沉积系统在低温 制备高质量外延硅锗薄膜方面前景广阔。

\section{参考文献:}

[1] PAUL D J. Silicon-germanium strained layer materials in microelectronics. Advanced Materials, 1999, 11(17): 191-204.

[2] CHANG S T, LIAO M H, LIN W K. Si/SiGe hetero-junction solar cell with optimization design and theoretical analysis. Thin Solid Films, 2011, 519(15): 5022-5025.

[3] HADI S A,HASHEMI P, NAYFEH A, et al. Thin film a-Si/c$\mathrm{Si}_{1-x} \mathrm{Ge}_{x} / \mathrm{c}-\mathrm{Si}$ heterojunction solar cells: design and material quality requirements, thin-film si cells. ECS Transactions, 2011, 41(4): 3-14.

[4] CANNON D D, LIU J, DANIELSON D T, et al. Germanium-rich silicon-germanium films epitaxially grown by ultrahigh vacuum chemical-vapor deposition directly on silicon substrates. Applied Physics Letters, 2007, 91(25): 252111.

[5] CHEN J X, ERNST F, HANSSON P O, et al. Liquid phase epitaxy of GeSi on $\{111\}$ Si substrates: lattice defect structure and electronic properties. Journal of Crystal Growth, 1992, 118(s 3-4): 452-460.

[6] CURRIEM T, SAMAVEDAM S B, LANGDO T A, et al. Controlling threading dislocation densities in $\mathrm{Ge}$ on $\mathrm{Si}$ using graded $\mathrm{SiGe}$ layers and chemical-mechanical polishing. Applied Physics Letters, 1998, 72(14): 1718-1720.

[7] LOH T H, NGUYEN H S, TUNG C H, et al. Ultrathin low temperature SiGe buffer for the growth of high quality Ge epilayer on $\mathrm{Si}(100)$ by ultrahigh vacuum chemical vapor deposition. Applied Physics Letters, 2007, 90(90): 092108-1-3.

[8] GUO L, ZHAO S, WANG J, et al. Fabrication of strained Ge film using a thin SiGe virtual substrate. Journal of Semiconductors, 2009, 30(9): 16-20.

[9] LOO R, SOURIAU L, ONG P, et al. Smooth and high quality epitaxial strained Ge grown on $\mathrm{SiGe}$ strain relaxed buffers with 70-85\% Ge. Journal of Crystal Growth, 2011, 324(1): 15-21.

[10] CHOI D, GE Y, HARRIS J S, et al. Low surface roughness and threading dislocation density Ge growth on Si (001). Journal of Crystal Growth, 2008, 310(18): 4273-4279.

[11] KIM H-W, SHIN K W, LEE G D, et al. High quality Ge epitaxial layers on Si by ultrahigh vacuum chemical vapor deposition. Thin Solid Films, 2009, 517(14): 3990-3994.
[12] YAMAMOTO M, HANNA J, MIYAUCHI M. New low pressure chemical vapor deposition technique for Ge crystalline thin films. Applied Physics Letter, 1993, 63(18): 2508-2510.

[13] YAMAMOTO M, TAKADA Y, HANNA J. Selective growth of Ge in $\mathrm{GeF}_{4}-\mathrm{Si}_{2} \mathrm{H}_{6}$ system. Applied Physics Letter, 1994, 64(25): 3467-3469.

[14] HANNA J, SHIMIZU K. Low-temperature growth of polycrystalline $\mathrm{Si}$ and Ge films by redox reactions of $\mathrm{Si}_{2} \mathrm{H}_{6}$ and $\mathrm{GeF}_{4}$. Journal of Organometallic Chemistry, 2000, 611(1): 531-536.

[15] TAO K, KUROSAWA Y, HANNA J. Low-temperature epitaxial growth of high quality $\mathrm{Si}_{1-x} \mathrm{Ge}_{x}(x \geqslant 0.99)$ films on $\mathrm{Si}(001)$ wafer by reactive thermal chemical vapor deposition. Applied Physics Letters, 2013, 102: 182109-1-5.

[16] NAYFEH A, CHUI C O, SARASWATK C, et al. Effects of hydrogen annealing on heteroepitaxial-Ge layers on $\mathrm{Si}$ : surface roughness and electrical quality. Applied Physics Letters, 2004, 85(14): 2815-2817.

[17] HARTMANN J M, ABBADIEA, BARNES J P, et al. Impact of the $\mathrm{H}_{2}$ anneal on the structural and optical properties of thin and thick Ge layers on $\mathrm{Si}$; low temperature surface passivation of $\mathrm{Ge}$ by $\mathrm{Si}$. Journal of Crystal Growth, 2010, 312(4): 532-541.

[18] KOBAYASHI S, NISHI Y, SARASWAT K C. Effect of isochronal hydrogen annealing on surface roughness and threading dislocation density of epitaxial Ge films grown on Si. Thin Solid Films, 2010, 518(6): S136-S139.

[19] LUAN H C, LIM D R, LEE K K, et al. High-quality Ge epilayers on Si with low threading-dislocation densities. Applied Physics Letters, 1999, 75(19): 2909-2911.

[20] PEZZOLI F, BONERA E, GRILLI E, et al. Raman spectroscopy determination of composition and strain in image heterostructures. Materials Science in Semiconductor Processing, 2008, 11(s 5-6): 279-284.

[21] SHAH V A, DOBBIE A, MYRONOV M, et al. High quality relaxed Ge layers grown directly on a $\mathrm{Si}(001)$ substrate. Solid-State Electronics, 2011, 62(1): 189-194.

[22] ZHOU Z, LI C, LAI H, et al. The influence of low-temperature Ge seed layer on growth of high-quality Ge epilayer on $\mathrm{Si}(100)$ by ultrahigh vacuum chemical vapor deposition. Journal of Crystal Growth, 2008, 310(10): 2508-2513.

[23] OLUBUYIDE O O, DANIELSON D T,KIMERLING L C, et al. Impact of seed layer on material quality of epitaxial germanium on silicon deposited by low pressure chemical vapor deposition. Thin Solid Films, 2006, 508(1): 14-19.

[24] HARTMANN J M, DAMLENCOURT J F, BOGUMILOWICZ Y, et al. Reduced pressure-chemical vapor deposition of intrinsic and doped ge layers on $\mathrm{Si}(001)$ for microelectronics and optoelectronics purposes. Journal of Crystal Growth, 2005, 274(1/2): 90-99.

[25] TAN Y H, TAN C S. Growth and characterization of germanium epitaxial film on silicon (001) using reduced pressure chemical vapor deposition. Thin Solid Films, 2012, 520(7): 2711-2716. 